\title{
Gestational Trophoblastic Neoplasm by AJCC v8 Stage
}

National Cancer Institute

\section{Source}

National Cancer Institute. Gestational Trophoblastic Neoplasm by A/CC v8 Stage. NCI

Thesaurus. Code C140033.

A term that refers to the staging of a gestational trophoblastic neoplasm of the placenta according to the American Joint Committee on Cancer, 8th edition. This classification applies to the following placental neoplasms: invasive hydatidiform mole,

choriocarcinoma, placental site trophoblastic tumor, and epithelioid trophoblastic tumor. Complete and partial hydatidiform tumors are not included in this classification. The current FIGO classification includes an anatomic stage designated by Roman numeral I, II, III, or IV, followed by the risk factor score expressed in Arabic numerals (e.g., Stage II: 4, Stage IV: 9). (from AJCC 8th Ed.) 